\title{
ACTIVIDAD FOTOCATALÍTICA DE PELÍCULAS DE TiO SENSIBILIZADAS CON COMPLEJOS METÁLICOS
}

\author{
Photocatalytic activity of TiO $_{2}$ films Sensitized With Metal complexes
}

César Augusto Quiñones Segura*

Diana Marcela Saavedra Morales**

Gina Paola Rocha Aranguren***

Recibido: 11 de enero de 2017

Aceptado: 25 de abril de 2017

\section{Resumen}

Se efectuó un análisis de las películas de $\mathrm{TiO}_{2}$ sensibilizadas con complejos metálicos de níquel y cobalto (II) contenidos en ligandos derivados del 3.5-dimetilpirazol. Se realizó un estudio preliminar del efecto del protocolo de limpieza de sustrato y de la temperatura de calcinación de las películas de $\mathrm{TiO}_{2}$ sin modificar. Se llevó a cabo la modificación de las películas con el método de dip coating variando el solvente utilizado en la dilución de los complejos y el tiempo del tratamiento térmico posterior a la modificación. Finalmente se evaluó la actividad fotocatalítica de las películas modificadas mediante la degradación de azul de metileno en presencia de radiación ultravioleta y luz visible.

Palabras clave: complejos metálicos, fotocatálisis, $\mathrm{TiO}_{2}$, sensibilización.

\section{Abstract}

It was done an analysis of $\mathrm{TiO}_{2}$ films sensitized with nickel and cobalt (II) metal complexes contained in ligands derived from 3.5-dimethylpyrazole. A preliminary study was carried out on the effect of the substrate cleaning protocol and the calcination temperature of unmodified $\mathrm{TiO}_{2}$ films. A modification of the films was carried out using the dip coating method, varying the solvent used in the dilution of the complexes and the time of the heat treatment after the modification. Finally, the photocatalytic activity of the modified films was evaluated by means of the degradation of methylene blue in the presence of ultraviolet radiation and visible light.

Keywords: metal complexes, photocatalysis, $\mathrm{TiO}_{2}$, sensitization.

\footnotetext{
* Químico, doctor en Química. Docente investigador, grupo de investigación Materiales Fotoactivos, Fundación Universidad de América. cesar.quiñones@profesores.uamerica.edu.co

** Estudiante coinvestigadora, grupo de investigación Materiales Fotoactivos Programa Ingeniería Química, Fundación Universidad de América. diana.saavedra@estudiantes.uamerica.edu.co

${ }^{* * *}$ Estudiante coinvestigadora, grupo de investigación Materiales Fotoactivos, Programa Ingeniería Química, Fundación Universidad de América. gina.rocha@ estudiantes.uamerica.edu.co
} 


\section{INTRODUCCIÓN}

En los últimos años la fotocatálisis ha sido utilizada en un amplio campo de investigaciones, principalmente en el área energética y ambiental. El objetivo de los procesos con fotocatálisis es la búsqueda y uso de materiales que conviertan energía solar en energía química para oxidar o reducir materiales, y de esta manera eliminar la presencia de contaminantes en el agua o el aire.

$\mathrm{E} 1 \mathrm{TiO}_{2}$ es uno de los fotocatalizadores más ampliamente usado y aplicado en fotocatálisis heterogénea, debido a su capacidad como oxidante para la descomposición de contaminantes orgánicos, larga durabilidad, bajo costo, estabilidad química y gran disponibilidad (Nakata y Fujishima, 2012). Sin embargo, la aplicación en procesos fotocatalíticos a gran escala está limitada por la necesidad de radiación UV para su activación, debido a que tiene un band gap de $2.32 \mathrm{eV}$, siendo esta la diferencia energética entre la parte superior de la banda de valencia y la parte inferior de la banda de conducción del semiconductor (Pedraza, 2005). Cabe destacar que, a pesar de hacer uso de luz solar como fuente de energía, solo es activo bajo radiación UV $(\lambda<390 \mathrm{~nm})$, lo que constituye menos del $5 \%$ de la intensidad del espectro de energía solar que llega a la tierra (Torres et ál., 2013), lo anterior representa una limitación, puesto que no se utilizan al máximo sus propiedades fotocatalíticas (Leguizamón et al., 2010). Según Mao et ál. (1998) y Hirano et ál. (2000), una alternativa que viene siendo explorada es emplear compuestos fotosensibilizadores, como colorantes y complejos metálicos, con el fin de extender el rango de fotorrespuesta del $\mathrm{TiO}_{2}$ a la región visible.

Para llevar a cabo el proceso fotocatalítico, se inicia acondicionando el sustrato sobre el cual se va a depositar la película de $\mathrm{TiO}_{2}$, en este caso el sustrato empleado es un vidrio de silicato; con el propósito de obtener una superficie limpia y libre de cualquier tipo de grasa o impureza que pueda estar adherida a su superficie y afecte la correcta fijación de la película se lleva a cabo un protocolo de limpieza. Posteriormente se realiza una suspensión de $\mathrm{TiO}_{2}$, polietilenglicol e isopropanol y se da inicio a la elaboración de la película de $\mathrm{TiO}_{2}$ mediante la técnica spin coating, la cual consiste en depositar sobre el centro de la superficie del sustrato una cantidad conocida de la suspensión, y luego aplicar fuerza centrífuga para que fluya radialmente hacia afuera, cubriendo de forma uniforme la totalidad del sustrato y finalmente se realiza un tratamiento térmico.

La sensibilización de las películas elaboradas anteriormente se realiza mediante la técnica de dip coating, al sumergir dichas películas en una solución que contiene los complejos metálicos [Cobis (N,N-3.5-dmpz)(Cl)2] y [Ni-bis(N,N-3.5dmpz)(NCS)2], disueltos en distintos solventes de acuerdo a su solubilidad.

En este sentido, fueron sensibilizadas películas de $\mathrm{TiO}_{2}$ con complejos de níquel y cobalto (II), contenidos en ligandos derivados de 3.5-dimetilpirazol (3.5-dmpz), para potenciar su actividad en luz visible y finalmente evaluar su actividad fotocatalítica mediante la degradación de azul de metileno (AM).

\section{Metodología}

\section{Elaboración de películas}

Las variables y parámetros a usar se establecieron según los proyectos realizados anteriormente en el grupo de investigación de materiales fotoactivos, con el fin de tener un seguimiento, estudio y control de las mismas. En principio las películas delgadas de $\mathrm{TiO}_{2}$ se hicieron mediante la técnica spin coating, posteriormente las películas fueron calcinadas a $550^{\circ} \mathrm{C}(\mathrm{T} 1), 600{ }^{\circ} \mathrm{C}(\mathrm{T} 2)$ y $650{ }^{\circ} \mathrm{C}$ (T3) para activar el $\mathrm{TiO}_{2}$, y eliminar los solventes que contiene el fotocatalizador, para conducir de esta forma a la sinterización de las partículas de $\mathrm{TiO}_{2}$ entre ellas y con el sustrato. 


\section{Sensibilización de películas}

La modificación de las películas de $\mathrm{TiO}_{2}$ se realizó con los complejos Co-bis(N,N-3,5-dmpz) $(\mathrm{Cl})_{2}(\mathrm{C} 1)$ y Ni-bis $(\mathrm{N}, \mathrm{N}-3.5 \mathrm{dmpz})(\mathrm{NCS})_{2}(\mathrm{C} 2)$ mediante la técnica conocida como dip coating, en la cual se sumergieron las películas de $\mathrm{TiO}_{2}$ durante un tiempo de 10 segundos en una solución del agente modificante.

Para el complejo C1 se realizó la disolución en metanol y Acetonitrilo (ACN), mientras que para el C2 en metanol y propanol dependiendo el tiempo de calcinación. Con el fin de obtener películas con propiedades fotocatalíticas y buena adherencia entre el complejo metálico y el $\mathrm{TiO}_{2}$, se realizó un tratamiento térmico posterior para dar lugar a la sinterización de las partículas, esto hace que se reduzca el volumen de la película y por lo tanto su espesor (Yu et ál., 2002). En este caso dicho tratamiento térmico se llevó a cabo variando el tiempo de calentamiento a 1 y 2 horas manteniendo la temperatura a $100^{\circ} \mathrm{C}$ y se evaluó el efecto de la duración del tratamiento en la actividad fotocatalítica de las películas sensibilizadas.

En adelante, para identificar las películas utilizadas en las degradaciones de azul de metileno se hará uso de una nomenclatura para hacer referencia al solvente modificante y el tiempo de tratamiento térmico. Para el $\mathrm{C} 1$ al metanol con 1 hora de calentamiento (M1), metanol con 2 horas (M2), ACN con 1 hora (M3) y ACN con 2 horas (M4), mientras que para las películas sensibilizadas con C2, al metanol con 1 hora (M5), metanol con 2 horas (M6), propanol con 1 hora (M7) y propanol con 2 horas de calentamiento (M8).

\section{Evaluación de la actividad fotocatalítica}

Se realizó una curva de calibración para el AM (figura 1) con el fin de llevar a cabo un seguimiento de la disminución de la concentración del colorante en función del tiempo; inicialmente se partió de una solución patrón de 50 ppm y a partir de esta se prepararon soluciones de 1 a 8 ppm. La medición se llevó a cabo en un espectrofotómetro a una longitud de $664 \mathrm{~nm}$, puesto que en esta medida el AM presentó su mayor absortividad.

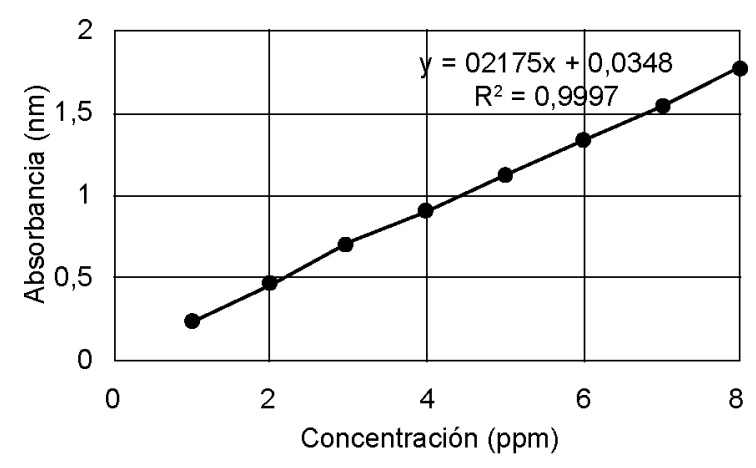

Figura 1. Curva de calibración del AM.

A partir de la ecuación de la recta presentada en la gráfica anterior, se puede determinar la concentración de AM para cualquier valor de absortividad. Para la evaluación de la actividad fotocatalítica de las películas se realizó un seguimiento por espectrofotometría de la degradación de AM bajo radiación UV y luz visible. 


\section{Resultados}

\section{Películas modificadas por dip coating}

Las películas modificadas con $\mathrm{C} 1$ presentaron una coloración azul intensa tanto en el momento de la deposición del complejo, como al finalizar el tratamiento térmico. Mientras que las películas modificadas con $\mathrm{C} 2$ presentaron color únicamente durante el proceso del dip coating, como se evidencia en las figuras 2 y 3 .

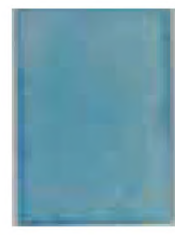

A) C1.M1

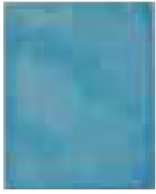

B) C1.M2

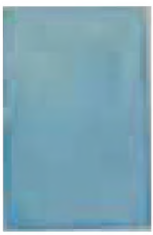

C) $\mathrm{C} 1 . \mathrm{M3}$

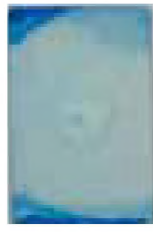

D) C1.M4

Figura 2. Película modificadas con $\left[\mathrm{Co}-\mathrm{bis}(\mathrm{N}, \mathrm{N}-3.5-\mathrm{dmpz})(\mathrm{Cl})_{2}\right]$.

Como se observa en la figura 2, las películas modificadas con el complejo $\mathrm{C} 1$ presentaron un color azul intenso. Se puede evidenciar que al variar los solventes modificantes y el tiempo de calentamiento con el fin de adherir el agente modificante a la película, no presentan variación de color. La película $\mathrm{D}$ correspondiente al modificante 4 ( $\mathrm{ACN}$ a $2 \mathrm{~h}$ ), presenta en los bordes de la película una tonalidad más oscura, una posible causa es que la película pudo haber estado sumergida en la dilución del complejo más del tiempo estipulado durante la técnica de dip coating.

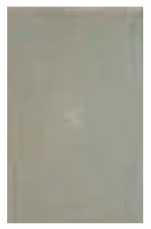

A) C2.M5

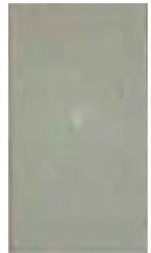

B) $\mathrm{C} 2 . \mathrm{M} 6$

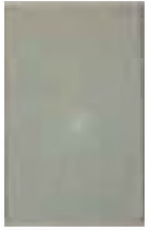

C) C2.M7

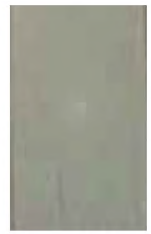

D) C1.M8

Figura 3. Películas modificadas con [Ni-bis(N,N-3,5 dmpz) (NCS) $)_{2}$.

En la figura 3, se evidencia que las películas modificadas con C2 no presentan cambios en la coloración de las películas de $\mathrm{TiO}_{2}$, a pesar de que al igual que en el caso anterior se hicieron variaciones y valoraciones cualitativas del solvente empleado en la dilución y del tiempo de calentamiento.

\section{Evaluación de la actividad fotocatalítica}

Para corroborar si efectivamente el protocolo de limpieza del sustrato y la temperatura de calcinación de las películas influyen en la actividad fotocatalítica del $\mathrm{TiO}_{2}$, se realizaron degradaciones de AM en luz visible y radiación UV (ver figura 4). 


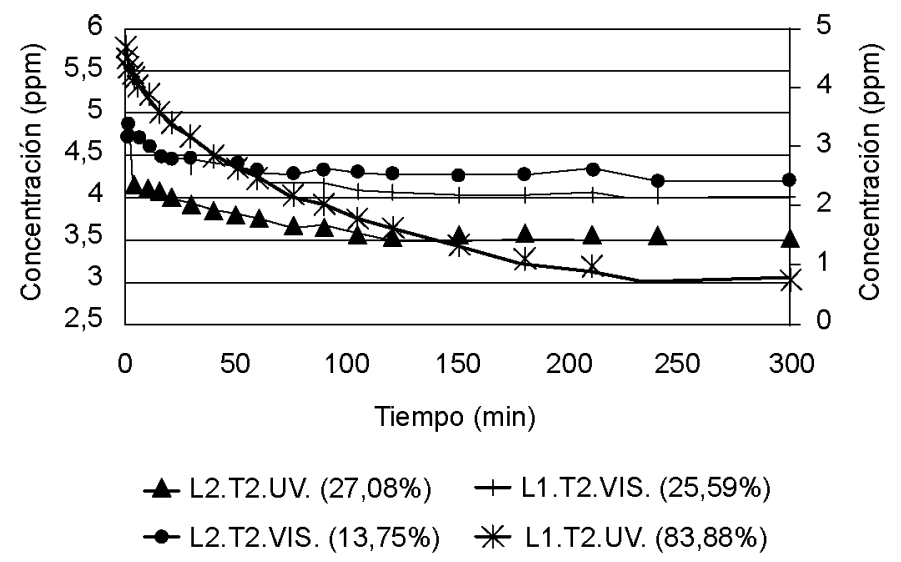

Figura 4. Degradación de AM para películas con protocolo de limpieza de sustrato 1 y 2.

En la figura 4 se observa que las películas de protocolo de lavado (L1) presentan una mejor degradación de AM en comparación con las películas de protocolo de lavado (L2), lo anterior puede deberse a que en el protocolo de limpieza de sustrato L1 el último solvente empleado es el etanol y para el protocolo L2 el ultimo solvente empleado es agua. Debido a que el etanol es más volátil que el agua, el protocolo L1 requiere cerca de dos minutos en el proceso de secado; mientras que en el proceso de secado del protocolo de limpieza L2 se requieren de 30 a 40 segundos más que en el anterior. Por ende, se aumenta la exposición del sustrato a partículas contaminantes presentes en el medio. Por esta razón, las siguientes degradaciones fueron realizadas con las películas L1.

En la figura 5 se muestran las curvas de degradación de $\mathrm{AM}$ con las películas de $\mathrm{TiO}_{2}$ calcinadas a diferentes temperaturas. Se puede observar que las películas degradadas en VIS en comparación con las UV presentan mayor concentración de azul de metileno al final del proceso y por ende, menores porcentajes de degradación, esto es debido a la baja actividad fotocatalítica que presenta el $\mathrm{TiO}_{2}$ en luz visible. La película L1.T2.UV presenta la mejor degradación de AM con un porcentaje de $83.88 \%$; adicionalmente se observa que a partir del minuto 150 la concentración del AM es aproximadamente constante, lo que indica que requiere un menor tiempo para la degradación con respecto a las demás películas tanto en UV como en VIS.

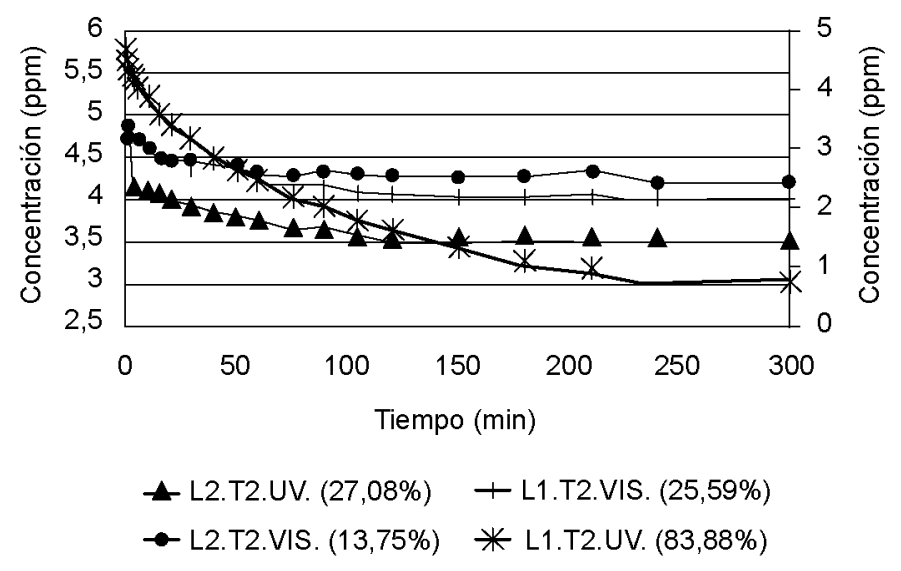

Figura 5. Degradación de AM para películas con variación de temperaturas. 
El aumento de la temperatura genera una disminución del band gap, del volumen y el tamaño de poro de los recubrimientos, por ende, Yu et ál. (2000) y Yu et ál. (2002) afirman que a temperaturas superiores a $600{ }^{\circ} \mathrm{C}$ pueden comenzar a aparecer partículas de rutilo lo que produce un colapso parcial de la porosidad, en donde dichos defectos de la red pueden actuar como centros de recombinación de los pares electrón-hueco ( $\mathrm{Li}$ et ál., 2004); por lo anterior, las películas calcinadas a $650^{\circ} \mathrm{C}$ fueron las que presentaron un menor porcentaje de degradación con respecto a las calcinadas a $550{ }^{\circ} \mathrm{C}$ y $600^{\circ} \mathrm{C}$.

Las degradaciones de $\mathrm{AM}$ bajo radiación UV de las películas de $\mathrm{TiO}_{2}$ modificadas con $\mathrm{C} 1$ se representan en la figura 6 .

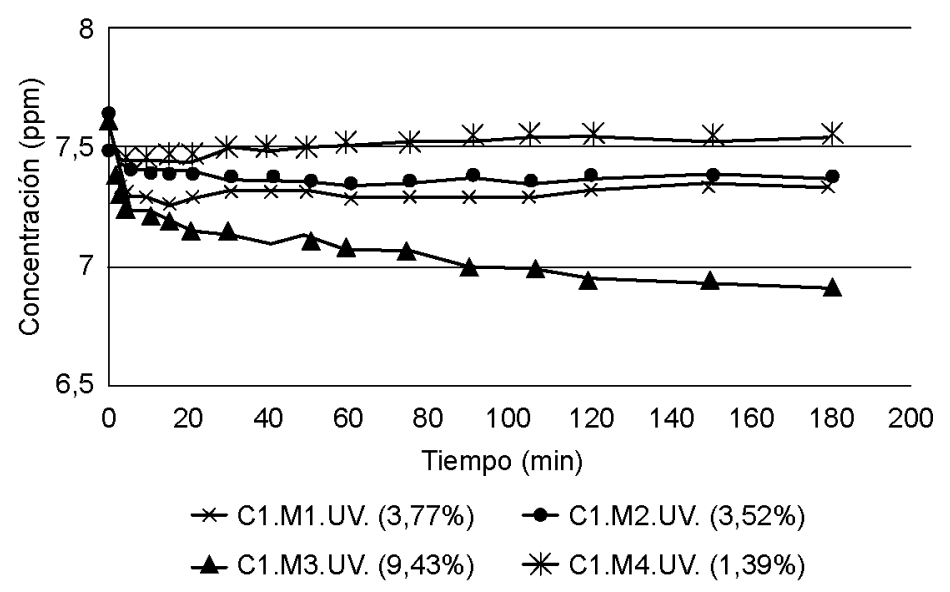

Figura 6. Degradación de AM para películas de $\left[\right.$ Co-bis $\left.(\mathrm{N}, \mathrm{N}-3.5-\mathrm{dmpz})(\mathrm{Cl})_{2}\right]$ en radiación UV

Se evidencia que las película C1.M3.UV, correspondiente a la modificada haciendo uso de ACN como solvente y con 1 hora de calentamiento, presentó el mayor porcentaje de degradación con un $9.43 \%$. Además, se evidencia que los mayores porcentajes de degradación $9.43 \%$ y $3.77 \%$ corresponden a las películas C1.M3.UV y C1.M1.UV, respectivamente, dichas películas fueron sometidas al tratamiento térmico por $1 \mathrm{~h}$. Lo anterior indica que el solvente de dilución del complejo y el tiempo de calcinación de las películas influye en la actividad fotocatalítica de las películas.

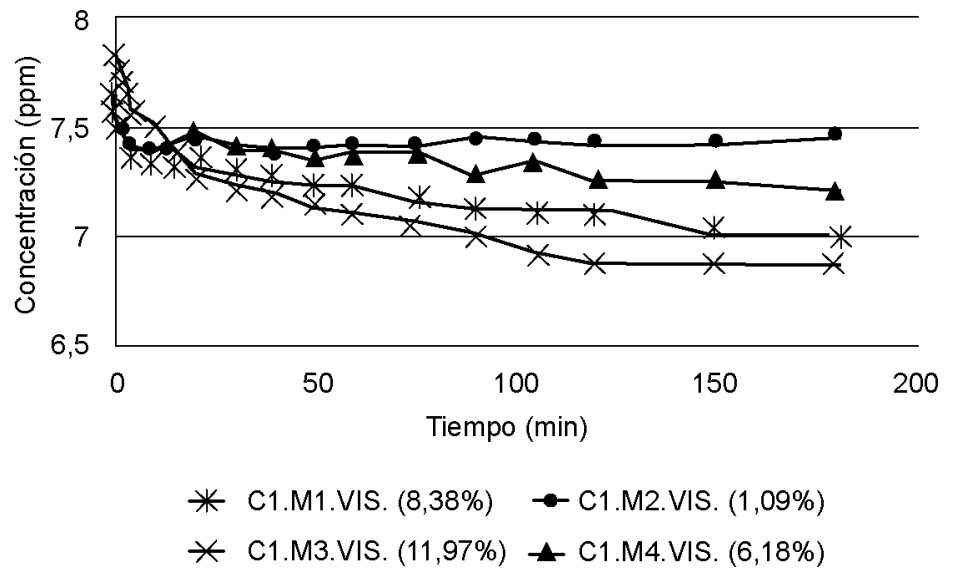

Figura 7. Degradación de AM para películas de [Co-bis $\left.(\mathrm{N}, \mathrm{N}-3.5-\mathrm{dmpz})(\mathrm{Cl})_{2}\right]$ en luz visible. 
En la figura anterior se observa nuevamente que la película que presenta la mejor degradación de AM (11,97\%) es C1.M3.VIS, es decir, la sensibilizada con el complejo disuelto en $\mathrm{ACN}$, con un tiempo de calentamiento de 1 hora. Al igual que en la gráfica 3, la película modificada con el complejo disuelto en metanol y con un tiempo de tratamiento térmico de 1 hora, es decir, la película C1.M1.VIS es la que presenta el segundo mayor porcentaje de degradación (8.38 \%).

El hecho de que las películas sensibilizadas con el complejo diluido en ACN presentaran los mayores porcentajes de degradación, tanto bajo radiación UV, como visible, puede deberse a que el complejo presenta un mayor grado de solvatación a medida que incrementa la polaridad del solvente en el que es diluido.

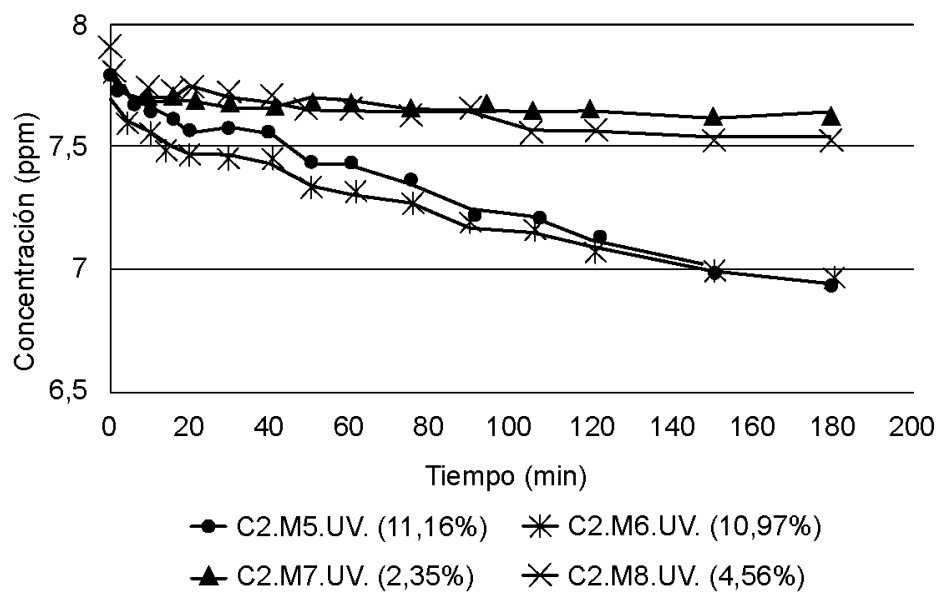

Figura 8. Degradación de AM para películas de $\left[\mathrm{Ni}-\mathrm{bis}(\mathrm{N}, \mathrm{N}-3.5 \mathrm{dmpz})(\mathrm{NCS})_{2}\right]$ en radiación UV.

En la figura 8 es notable que las películas modificadas con el complejo disuelto en metanol tienen mayor actividad fotocatalitica que las modificadas con el complejo disuelto en propanol. Confirmando que a mayor polaridad del solvente se obtiene una mejor degradación de AM. La película C2.M5.UV con un $11.16 \%$ de degradación es la que mayor porcentaje de degradación presenta, la cual es nuevamente la película calentada a 1 hora.

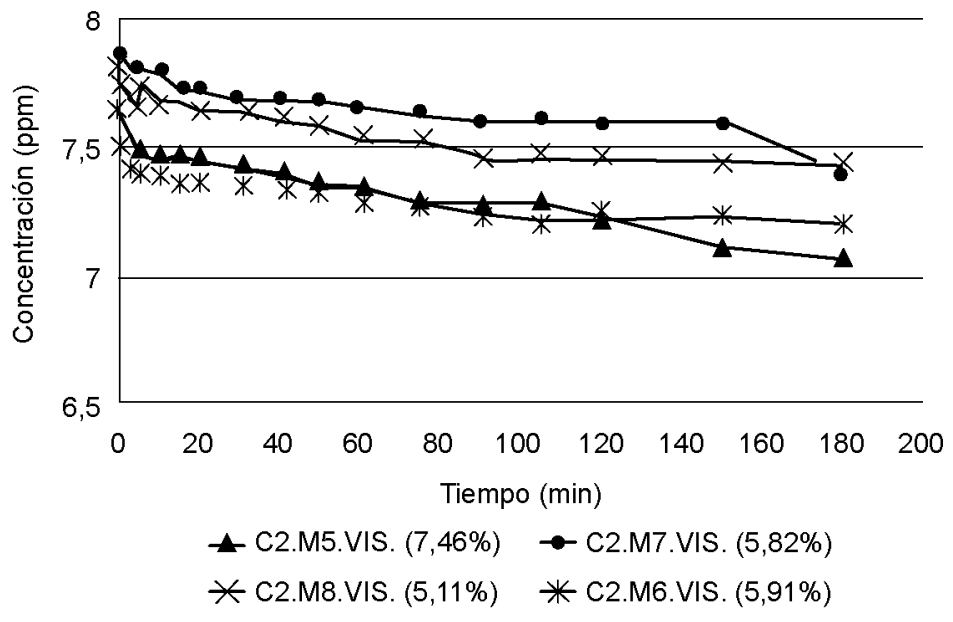

Figura 9. Degradación de AM para películas de $\left[\mathrm{Ni}-\mathrm{bis}(\mathrm{N}, \mathrm{N}-3.5 \mathrm{dmpz})(\mathrm{NCS})_{2}\right]$ en luz visible. 
En esta figura, nuevamente las películas sensibilizadas con el complejo disuelto en metanol C2.M5.VIS y C2.M6.VIS presentan los mayores porcentajes de degradación. La película C2.M5.VIS cuyo tiempo de calentamiento fue de 1 hora alcanzó un porcentaje de degradación de $7.46 \%$.

\section{Difracción de rayos $X$}

Con el fin de verificar el estado cristalino que presentan las películas de $\mathrm{TiO}_{2}$ modificadas luego del tratamiento térmico, se lleva a cabo una caracterización estructural mediante este análisis. Para establecer la fase del $\mathrm{TiO}_{2}$ predominante en las películas se toman como base los resultados de la caracterización por DRX de las fases anatasa y rutilo reportadas en la literatura (ver figura 10).

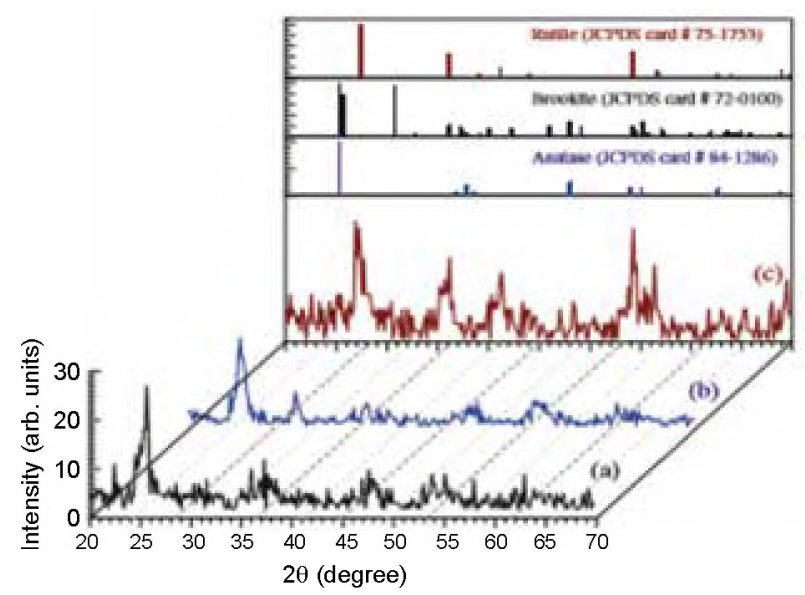

Figura 10. Patrones de difracción de rayos $X$ de muestras de $\mathrm{TiO}_{2}$.

Fuente: El-Sherbiny, Morsy, Samir y Fouad (2014).

En la figura 10 , el pico de difracción a 20 de $25^{\circ}$ es atribuido a la estructura cristalográfica de la fase anatasa, mientras que la fase rutilo presenta este pico a $28^{\circ}$ y en el caso de la brookita uno de los picos de mayor intensidad se presenta alrededor de los $32^{\circ}$ (E1-Sherbiny et ál., 2014). En las figuras 4 y 5 se hará uso de la nomenclatura de la tabla 1.

Tabla 1. Difractograma de las películas de $\mathrm{TiO}_{2}$ modificadas con [Co-bis $\left.(\mathrm{N}, \mathrm{N}-3.5-\mathrm{dmpz})(\mathrm{Cl})_{2}\right]$

\begin{tabular}{cl}
\hline Símbolo & Descripción \\
\hline A & Anatasa \\
\hline B & Brookita \\
\hline
\end{tabular}

En la figura 11 se puede observar que al modificar las películas de $\mathrm{TiO}_{2}$ con el $\mathrm{C} 1$ disuelto en distintos solventes y a distinta duración del tratamiento térmico, a 25 grados se presenta un pico de alta intensidad, el cual según la figura 24 corresponde $\mathrm{al} \mathrm{TiO}_{2}$ en fase anatasa. Lo que nos muestra que la modificación no hizo un cambio a nivel estructural del $\mathrm{TiO}_{2}$. 


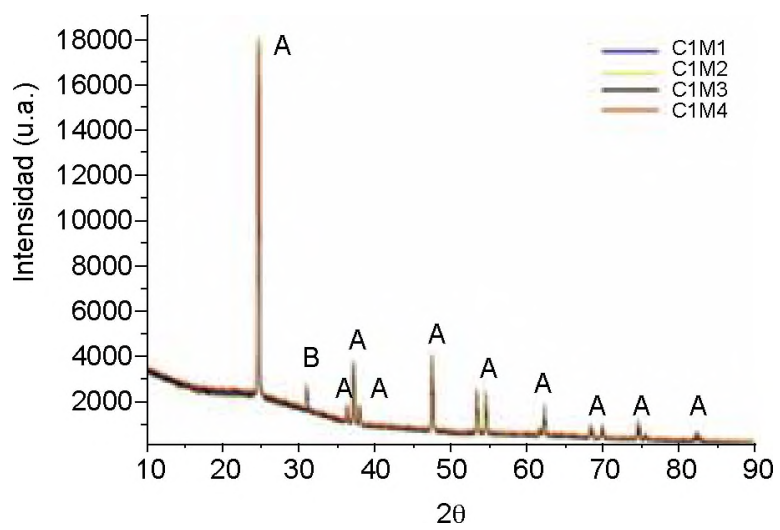

Figura 11. Difractograma de las películas de $\mathrm{TiO}_{2}$ modificadas con [Co-bis $\left.(\mathrm{n}, \mathrm{n} \cdot 3,5-\mathrm{dmpz})(\mathrm{Cl})_{2}\right]$.

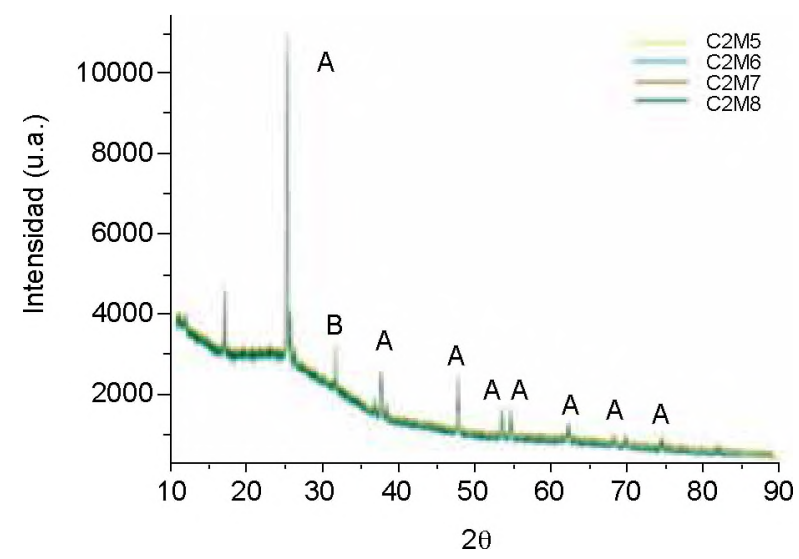

Figura 12. Difractograma de las películas de $\mathrm{TiO}_{2}$ modificadas con [Ni-bis(N,N-3.5-dmpz)(NCS) ].

En la figura 12, al igual que en el caso anterior, se observa que la modificación no alteró la estructura del $\mathrm{TiO}_{2}$, ya que se encuentran los picos que caracterizan $\mathrm{al}_{\mathrm{TiO}}$ en fase anatasa y el pico de mayor intensidad se presenta nuevamente a 25 grados.

\section{ANÁLISIS}

Además, en entornos acuosos la adsorción de colorantes se debe principalmente a la interacción electrostática con la superficie del $\mathrm{TiO}_{2}$, puesto que depende del $\mathrm{pH}$ de la solución. Se conoce que el punto isoeléctrico del $\mathrm{TiO}_{2}$ es a un $\mathrm{pH}=6$, por lo cual debe trabajarse a un $\mathrm{pH}$ diferente a este; es decir, para valores de $\mathrm{pH}$ mayores a 6 el $\mathrm{TiO}_{2}$ se carga de forma negativa (Park et ál., 2016) y repele electrostáticamente a los grupos de anclaje del ligando (ácido carboxílico, ácido sulfónico, ácido fosfonico, etc.).

Lo anterior es una posible causa de la baja actividad fotocatalítica que presentaron las películas de $\mathrm{TiO}_{2}$ modificadas con $\mathrm{C} 1$ y $\mathrm{C} 2$, pues los solventes (acetonitrilo, metanol y propanol) empleados en la modificación tienen un $\mathrm{pH}$ neutro; lo que pudo causar que el $\mathrm{TiO}_{2}$ y el sensibilizante se repelan electrostáticamente disminuyendo la eficiencia de la sensibilización. Cabe resaltar que el uso de dichos solventes se debió a la solubilidad que presentaron los complejos de [Co-bis $\left.(\mathrm{N}, \mathrm{N}-3.5-\mathrm{dmpz})(\mathrm{Cl})_{2}\right]$ y $\left[\mathrm{Ni}-\mathrm{bis}(\mathrm{N}, \mathrm{N}-3.5 \mathrm{dmpz})(\mathrm{NCS})_{2}\right]$ en los mismos. 
Otro factor que posiblemente afecta la estabilidad de los complejos es que en la adsorción del colorante sobre la superficie del $\mathrm{TiO}_{2}$ podría haber competitividad entre el soluto (complejo metálico) y el AM, para ocupar los sitios activos en la superficie del catalizador $\left(\mathrm{TiO}_{2}\right)$; por lo cual, el sistema se trataría como una reacción de adsorción-desorción superficial (Leguizamón et ál., 2010).

\section{Comentarios}

a. Se determinó que el protocolo de limpieza del sustrato influye en la adherencia del $\mathrm{TiO}_{2}$ al sustrato, debido a las volatilidades de los solventes empleados y por ende afecta su desempeño en la degradación del azul de metileno. Las películas depositadas sobre el sustrato lavado con L1 obtuvieron porcentajes de degradación de $83.88 \%$ y $25.59 \%$ bajo radiación UV y VIS, respectivamente, mientras que las películas depositadas sobre el sustrato lavado con L2 presentaron porcentajes de degradación de $27.08 \%$ en UV y $13.75 \%$ en VIS.

b. La adherencia del $\mathrm{TiO}_{2}$ al sustrato se ve afectada por la temperatura de calcinación y presentan mayor adherencia a una temperatura de calcinación de $650^{\circ} \mathrm{C}$ por el efecto de sinterización. La película de $\mathrm{TiO}_{2}$ que mejor actividad fotocatalítica presenta es a $600{ }^{\circ} \mathrm{C}$, con un porcentaje de degradación de azul de metileno de 20.79 \% bajo luz visible y $90.87 \%$ bajo radiación UV a partir del minuto 120.

c. La sensibilización del $\mathrm{TiO}_{2}$ utilizando el complejo $\left[\mathrm{Ni}\right.$-bis $\left.(\mathrm{N}, \mathrm{N}-3,5 \mathrm{dmpz})(\mathrm{SCN})_{2}\right]$ logró una degradación de azul metileno de $11.16 \%$ bajo radiación UV y de $7.46 \%$ en luz visible a los 180 minutos.

d. En 180 minutos las películas sensibilizadas con [Co-bis $(\mathrm{N}, \mathrm{N}-3.5-\mathrm{dmpz})(\mathrm{Cl})_{2}$ ] obtuvieron porcentajes de degradación de hasta $9.43 \%$ en radiación UV y de $11.97 \%$ en luz visible.

e. El solvente con el que se realiza la dilución de los complejos metálicos afecta los porcentajes de degradación de azul metileno debido a que los solventes que tienen mayor polaridad y constante dieléctrica, favorecen la actividad fotocatalítica. Para el complejo [Co-bis $\left.(\mathrm{N}, \mathrm{N}-3.5-\mathrm{dmpz})(\mathrm{Cl})_{2}\right]$ el solvente más polar es acetonitrilo y presento un porcentaje de degradación de $9.43 \%$ bajo radiación UV y $11.97 \%$ en VIS, mientras que en el caso del complejo [Ni-bis(N,N-3.5 dmpz) $\left.(\mathrm{SCN})_{2}\right]$ se obtuvo un porcentaje de degradación de $11.16 \%$ en UV y $7.46 \%$ en VIS haciendo uso de metanol, es nuevamente el solvente más polar .

\section{REFERENCIAS}

El-Sherbiny, S., Morsy, F., Samir, M., y Fouad, O. (2014). Synthesis, characterization and application of $\mathrm{TiO} 2$ nanopowders as special paper coating pigment. Applied Nanoscience, 4(3), 305-313.

González-Púmariega, M., Tamayo, M., y Sánchez-Lamar, Á. (2009) La radiación ultravioleta. Su efecto dañino y consecuencias para la salud humana. Theoria, 18(2), 69-80.

Hirano, K., Suzuki, E., Ishikawa, A., Moroy, T., Shiroishi, H., y Kaneko, K. (2000). Sensization of $\mathrm{TiO}_{2}$ particles by dyes to achieve $\mathrm{H}$ evolution by visible light. Journal of Photochemistry and Photobiology A: Chemistry, 136(3), 157-161.

Leguizamón, J., Quiñones, C., Espinosa, H., y Sarria, V. (2010). $\mathrm{TiO}_{2}$ photosensibilization with a commercial dye for photodegradation of organic water pollutants. Revista UDCA Actualidad É Divulgación Cientifica, 13(2) 185-190. 
Li, Y., Lee, N., Lee, E., y Kim, S. (2004). The characterization and photocatalytic properties of mesoporous rutile $\mathrm{TiO}_{2}$ powder synthesized through self-assembly of nano crystals. Chemical physics letters, 389(1), 124-128.

Mao, H., Deng, H., Li, H., Shen, Y., Lu, Z., y Xu, H.(1998) Photosensititaztion of $\mathrm{TiO}_{2}$ semiconductor with porphyrin. Journal of Photochemistry and Photobiology A: Chemistry, 114(3), 209-212.

Nakata, K., y Fujishima, A. (2012). $\mathrm{TiO}_{2}$ photocatalysis: design and applications. Journal of Photochemistry and Photobiology C: Photochemistry Reviews, 13(3), 169-189.

Pedraza, J. (2005). Estudio de la sensibilización de TiO2 con tetrasulfoftalocianinas metálicas en la oxidación fotocatalitica de ciclobexeno (tesis de maestría). Maestría en Química, Universidad Industrial de Santander, Bucaramanga, Colombia.

Park, H., KIM, H., Moon, G., y Choi, W. (2016). Photoinduced charge transfer processes in solar photocatalysis based on modifi ed $\mathrm{TiO}_{2}$. Energy E Environmental Science, 9(2), 411-433.

García, J. (s. f.). Formación de complejos. Recuperado de http://www.academia.edu/25485506/Formacion_de_complejos

Torres, J., Carriazo, J., y Sanabria, N. (2013). Síntesis y caracterización de bentonita modificada con especies de $\mathrm{TiO}_{2}$ y Fe-TiO $\mathrm{T}_{2}$ obtenidas de la mineral ilmenita. Revista Colombiana de Quimica, 42(1), 86-100.

Yu, J., Zhao, X., y Zhao, Q. (2000). Effect of film thickness on the grain size and photocatalytic activity of the sol-gel derived nanometer $\mathrm{TiO}_{2}$ thin films. Journal of Materials Science Letters, 19(12), 1015-1017.

Yu, J., Yu, J., Ho, W., y Jiang, Z. (2000). Effects of calcination temperature on the photocatalytic activity and photo-induced super-hydrophilicity of mesoporous $\mathrm{TiO}_{2}$ thin films. New Journal of Chemistry, 26(5), 607-613. 
